\title{
MODÉLISATION DE LA TRANSFORMATION POLYMORPHIQUE DU FER HOLTZER
}

\author{
P.L. HEREIL et F. DOUBAX \\ DGA/Centre d'Études de Gramat, F-46500 Gramat, France
}

\begin{abstract}
Résumé : Les transformations polymorphiques directes et inverses du fer Holtzer ont été observées au moyen d'essais d'impact de plaques et de l'interférométrie de vitesse. On présente un modéle de transformation thermodynamiquement cohérent dans lequel les variables indépendantes sont la contrainte moyenne et la température. Les comparaisons entre les diagrammes de vitesse expérimentaux et calculés par simulation numérique montrent les potentialités de cette modélisation.
\end{abstract}

\begin{abstract}
The direct and reverse polymorphic phase transitions of Holtzer iron were observed using plate impact experiments and velocity interferometric techniques. Thermodynamic consistent two-phases equation of state is presented with mean stress and temperature as independant variables. Confidence in the model is demonstrated by the comparisons between experimental and calculated velocity profiles.
\end{abstract}

\section{I - INTRODUCTION}

Depuis l'observation par Bancroft et al. /1/de la transformation polymorphique $(\alpha->\varepsilon)$ du fer sous choc pour une contrainte voisine de $130 \mathrm{kbar}$, cette singularité de comportement a fait l'objet de nombreuses études tant expérimentales que théoriques. Les résultats expérimentaux $/ 2 /, / 3 /, / 4 /, / 5 /$ représentés sur les figures 1 et 2 ont d'une part mis en évidence un hystérésis entre les transformations directes et inverses et ont d'autre part montré que ces transformations ne s'effectuaient pas dans des conditions d'équilibre thermodynamique. Les études théoriques $/ 6 /, / 7 /, / 8 /$ ont été consacrées à la modélisation des processus de transformation polymorphique à partir de la théorie des changements de phase. L'objectif du travail présenté dans cet article est de développer un modèle de transformation polymorphique du fer qui soit thermodynamiquement cohérent et qui reproduise le comportement de ce matériau jusqu'à une contrainte de 500 kbar.

\section{II - MODELE DE TRANSFORMATION POLYMORPHIQUE}

La caractérisation d'une transformation polymorphique nécessite d'élaborer les équations constitutives de chaque phase ainsi que celles correspondant au mélange des deux phases. Le point essentiel d'une telle modélisation réside dans la correspondance entre les équations constitutives des deux phases par l'intermédiaire des relations thermodynamiques caractérisant la transformation. De ce fait, il est indispensable pour obtenir un modèle de transformation thermodynamiquement cohérent, de s'assurer de la cohérence thermodynamique des équations constitutives de chaque phase ainsi que de celles caractérisant le mélange. 


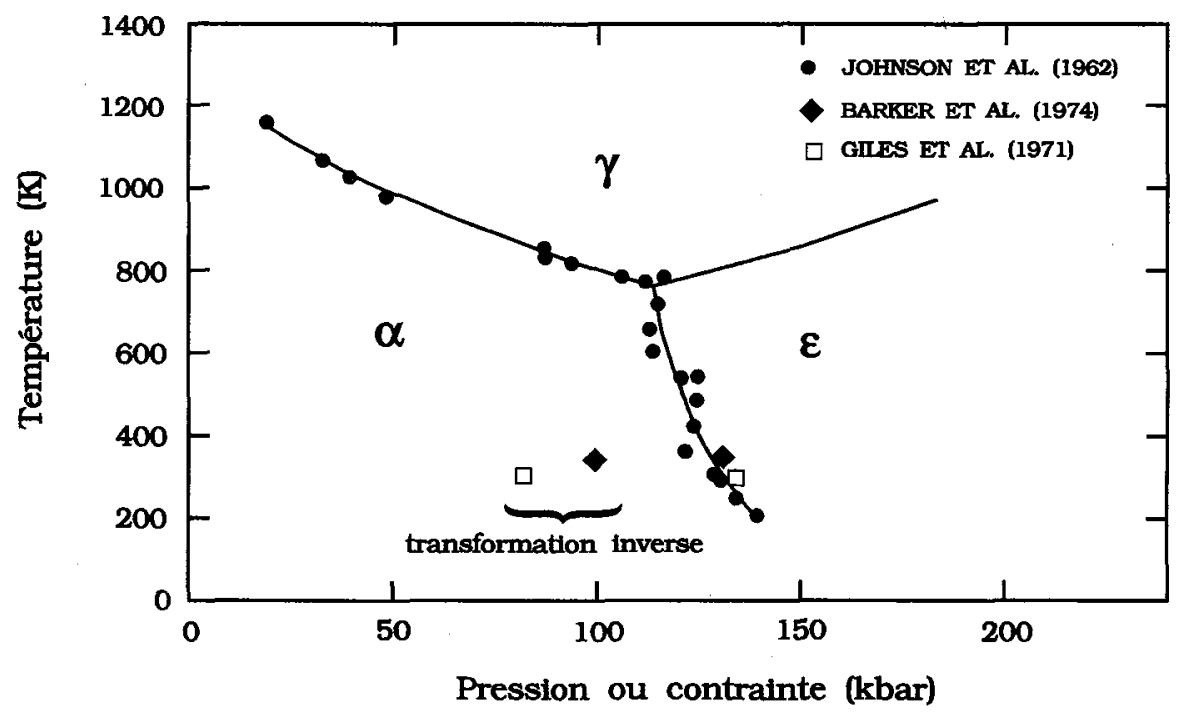

Figure 1

Diagramme de changement de phase du fer

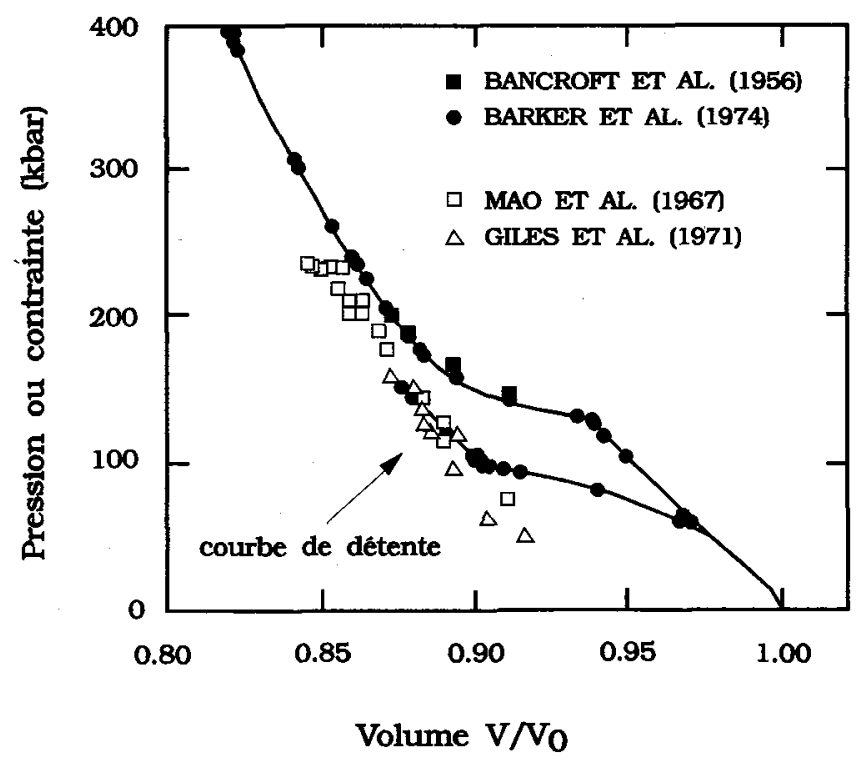

Figure 2

Représentation des transformations polymorphiques directes et inverses du fer. 


\section{II-1 - Equations constitutives des phases}

L'équation constitutive thermodynamiquement cohérente utilisée pour décrire le comportement de chaque phase repose sur une formulation établie par Cowperthwaite/9/et Johnson et al./10/et dans laquelle la chaleur spécifique $\mathrm{C}_{\mathrm{v}}$ ainsi que le rapport $\Gamma / \mathrm{V}$ où $\Gamma$ est le coefficient de Grüneisen et $\mathrm{V}$ le volume spécifique, sont supposés constants. Cette formulation présente l'avantage d'une relative simplicité tout en restant compatible avec les données expérimentales. Nous avons choisi de définir cette équation constitutive pour les phases $\alpha$ et $\varepsilon$ à partir de la relation de Murnaghan :

$$
\mathrm{K}=\mathrm{K}_{0}+\mathrm{n}_{0}\left(\mathrm{P}-\mathrm{P}_{0}\right)
$$

où $\mathrm{P}$ représente la contrainte moyenne, $\mathrm{K}$ le module de compression isotrope à température constante et $\mathrm{n}_{0}$ une constante. Ces deux équations constitutives ne sont pas complètement découplées, mais dépendent l'une de l'autre par l'intermédiaire de la variation de volume et de la chaleur latente associées à la transformation polymorphique. Cette dépendance est assurée dans notre modélisation, par l'égalité des énergies libres de Gibbs des deux phases sur la ligne de transformation polymorphique.

\section{II-2 - Equations constitutives du mélange}

L'élaboration des équations constitutives caractérisant le mélange s'effectue à partir de la théorie des changements de phase /11/ qui repose sur les hypothèses suivantes : a) la contrainte moyenne $\mathrm{P}$ et la température $T$ sont identiques pour les deux phases, b) l'énergie d'interface entre les deux phases est négligeable. Si on désigne par $\lambda$ la fraction massique de la seconde phase et par les indices 1 et 2 les variables correspondant respectivement à la première et à la seconde phase, les hypothèses ci-dessus entrainent les relations pour le volume spécifique $\mathrm{V}$ et l'énergie interne spécifique $\mathrm{E}$ :

qui aprés différenciation se réécrivent :

$$
\begin{aligned}
& V=(1-\lambda) V_{1}+\lambda V_{2} \\
& E=(1-\lambda) E_{1}+\lambda E_{2}
\end{aligned}
$$

$$
\begin{aligned}
\mathrm{dV} & =(1-\lambda) \mathrm{dV}_{1}+\lambda \mathrm{dV}_{2}+\left(\mathrm{V}_{2}-\mathrm{V}_{1}\right) \mathrm{d} \lambda \\
\mathrm{dE} & =(1-\lambda) \mathrm{dE}_{1}+\lambda \mathrm{dE}_{2}+\left(\mathrm{E}_{2}-\mathrm{E}_{1}\right) \mathrm{d} \lambda
\end{aligned}
$$

Les équations constitutives de chacune des deux phases établies dans le paragraphe précédent, permettent d'expliciter les quantités $d V_{1}, d V_{2}, d E_{1}$ et $d_{2}$ en fonction des variables $P$ et $T$. En introduisant de plus la conservation de l'énergie les relations ci-dessus aboutissent à un système d'équations qui aprés inversion se réécrit :

$$
\begin{aligned}
& d P=a_{11} d V+a_{12} d \lambda \\
& d T=a_{21} d V+a_{22} d \lambda
\end{aligned}
$$

où les coefficients $a_{i j}(i, j=1,2)$ sont des fonctions de $P, T$ et $\lambda$.

Compte-tenu des résultats expérimentaux présentés sur les figures 1 et 2 qui montrent, rappelons-le, que la transformation polymorphique du fer est caractérisée par un processus gradué et par un hystérésis, nous avons choisi de reproduire l'évolution de la transformation directe par une formulation proposé par Forbes $/ 8 /$ :

$$
\lambda_{\text {eq }}=1-\exp \left[\beta\left(\mathrm{G}_{1}-\mathrm{G}_{2}-\mathrm{A}\right)\right]
$$

où $\beta$ et $A$ sont des constantes traduisant respectivement le processus gradué et l'hystéréis évoqués ci-avant et où $G_{1}$ et $G_{2}$ correspondent aux énergies libres de Gibbs de la première et de la seconde phase. De même, la transformation inverse a été modélisée par la relation :

$$
\lambda_{\text {eq }}=\exp \left[\beta\left(\mathrm{G}_{1}-\mathrm{G}_{2}-\mathrm{A}\right)\right]
$$

L'objectif ultérieur de ce travail étant d'intégrer le modèle de transformation dans les logiciels de calcul bidimensionnels et tridimensionnels, nous n'avons donc pas considéré de cinétique de transformation car les temps caractéristiques de cette cinétique ne sont pas pour l'instant compatibles avec les pas de temps utilisés dans ces logiciels. De plus, nous avons supposé que la transition élastique-plastique du fer étudié pouvait être reproduite par un critère d'écoulement parfaitement plastique de type Von-Mises. 
Tableau I

Valeurs des paramètres caractéristiques de la phase $\alpha$ et de la phase $\varepsilon$ du fer.

\begin{tabular}{lcc}
\hline & phase $\alpha$ & phase $\varepsilon$ \\
\hline $\mathrm{K}_{0}(\mathrm{kbar})$ & 1550 & 2100 \\
$\mathrm{n}_{0}$ & 4.379 & 6.0 \\
$\Gamma$ & 1.735 & 2.0 \\
$\mathrm{C}_{\mathrm{v}}(\mathrm{J} / \mathrm{kg} / \mathrm{K})$ & 444.7 & 446.6 \\
\hline
\end{tabular}

\section{III - RESULTATS}

\section{III-1 - Essais d'impact de plaques}

Les caractéristiques du fer Holtzer faisant l'objet de cette caractérisation ont été publiées dans une étude précédente $/ 12 /$. Nous retiendrons seulement pour cette étude les valeurs de la densité $\rho_{0}=7.89 \mathrm{~g} / \mathrm{cm} 3$ et des vitesses de propagation longitudinale $\mathrm{C}_{\mathrm{L}}=5950 \mathrm{~m} / \mathrm{s}$ et transversale $\mathrm{C}_{\mathrm{s}}=3250 \mathrm{~m} / \mathrm{s}$. Les configurations expérimentales ainsi que les diagrammes de vitesse obtenus au cours des deux essais d'impact de plaques sont représentés sur les figures 3 et 4 .

Le diagramme de vitesse de surface libre correspondant au premier essai (essai 23) présente des caractéristiques identiques à celles déjà observées par Barker et Hollenbach / $3 /$ sur un fer Armco. Il est composé d'un précurseur élastique qui précède l'onde plastique correspondant à la phase $\alpha$ puis celle correspondant à la phase $\varepsilon$. Les perturbations que l'on observe au début de ces deux ondes résultent de la réflexion du précurseur élastique sur la surface libre de l'échantillon. Le dernier saut de vitesse apparaissant sur le diagramme correspond à la réflexion de l'onde $\alpha$ sur une discontinuité créée à proximité de la surface libre, discontinuité qui résulte de l'interaction entre l'onde $\alpha$ réfléchie sur la surface libre et l'onde $\varepsilon$ incidente.

La configuration expérimentale du second essai (essai 48) a été dimensionnée afin de mettre en évidence la transformation polymorphique inverse du fer. Le diagramme de vitesse correspondant à cet essai qui est représenté sur la figure 4, montre en effet une onde de compression précédée par le précurseur élastique, puis un palier de vitesse et enfin une onde de détente composée de trois pentes reflétant la transformation polymorphique inverse.

\section{III-2 - Comparaisons expérience-calcul}

Le modèle de transformation polymorphique présenté précédemment a été implanté dans un logiciel unidimensionnel lagrangien traitant la propagation des ondes. Les valeurs des paramètres caractéristiques des phases $\alpha$ et $\varepsilon$ sont regroupées dans le tableau I. Ces valeurs ont été déterminées soit à partir des données expérimentales de la littérature, soit par des ajustements successifs entre les diagrammes de vitesse calculés par simulation numérique et les diagrammes expérimentaux mesurés au cours des essais 23 et 48 . Les comparaisons entre les diagrammes calculés et les diagrammes expérimentaux représentées sur les figures 3 et 4 montrent que la modélisation précédente permet de restituer la chronométrie et la structure des ondes à la fois pour les transformations directes et inverses. Néanmoins, on observe deux écarts significatifs entre le calcul et l'expérience. En premier lieu, on constate sur la figure 3, que la valeur de vitesse calculée au maximum de la phase $\alpha$ est plus faible que la valeur expérimentale. Ceci est dû au fait que la modélisation ne permet pas de restituer l'amortissement de l'onde $\alpha$ lié à la cinétique de transformation. En effet, si on se réfère à la loi d'amortissement de cette onde en fonction de la distance de propagation et de la contrainte maximale $/ 3 /$, on constate que l'écart observé est cohérent avec les conditions de l'essai 23 , à savoir une 


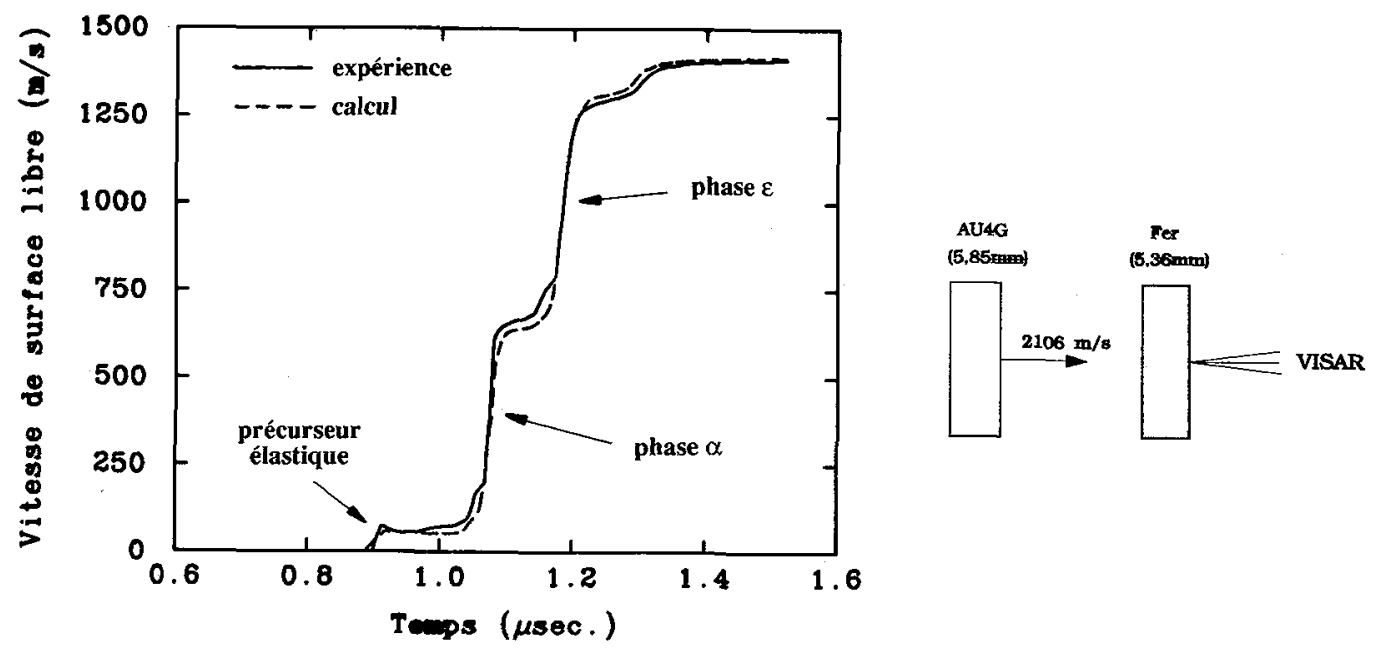

Figure 3

Comparaison entre les diagrammes de vitesse mesurés au cours de l'essai 23 et les diagrammes calculés par simulation numérique.

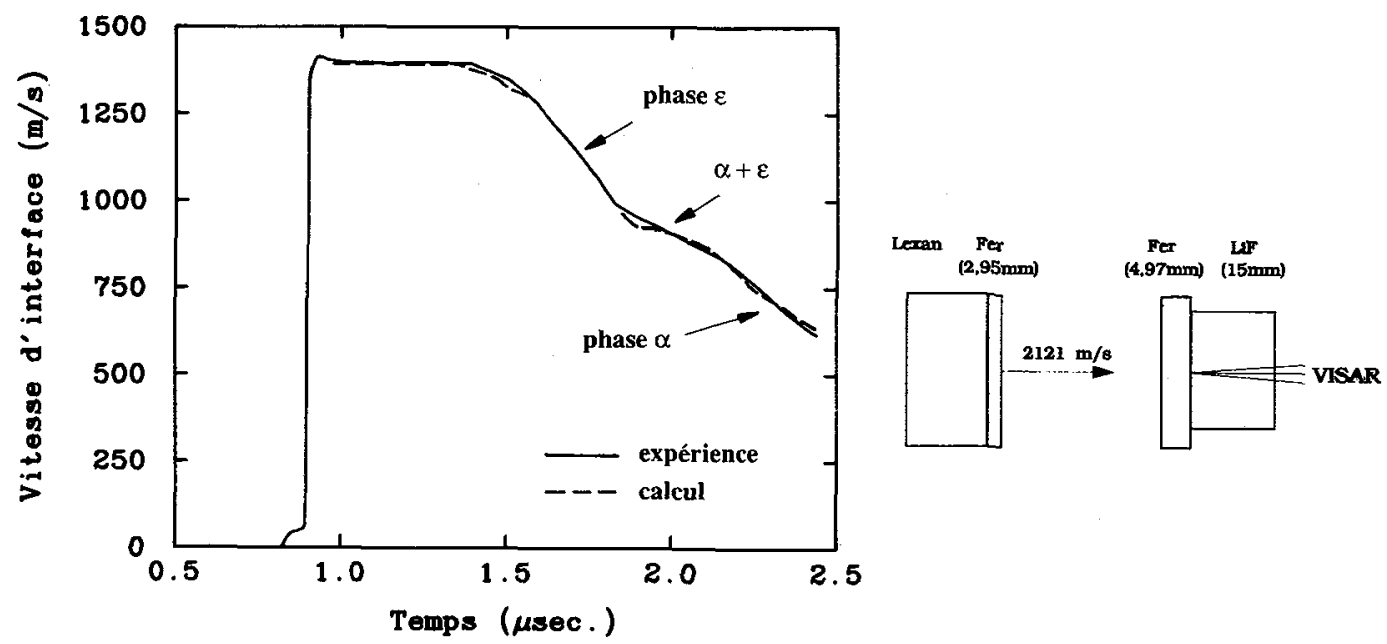

Figure 4

Comparaison entre les diagrammes de vitesse mesurés au cours de l'essai 48 et les diagrammes calculés par simulation numérique. 
distance de propagation de $5.36 \mathrm{~mm}$ et une contrainte maximum de $270 \mathrm{kbar}$. En second lieu, la comparaison illustrée sur la figure 4 montre que les pentes correspondant à la transformation inverse ne sont pas correctement calculées. Cet écart semblerait provenir d'une caractérisation inadéquate de la transformation inverse. Des expériences complémentaires en cours de réalisation au CEG devraient permettre de lever le doute sur ce dernier point .La particularité essentielle de cette modélisation concerne la valeur établie pour le paramètre $\mathrm{A}$, à savoir $\mathrm{A}=0$. Cette valeur nulle signifie en effet que l'hystérésis observé dans le plan contrainte-volume (figure 2) ne correspond pas à un "retard" des transitions directes et inverses par rapport à la ligne de transformation polymorphique. Cet hystérésis n'est donc lié qu'à la production d'entropie au cours du cycle compression-détente. Ce résultat, qui n'est pas en accord avec les valeurs expérimentales déterminées au cours d'essais quasi-statiques /4/ doit toutefois être infirmé ou confirmé par des essais complémentaires. Il serait dans ce sens, particulierement judicieux de réaliser des essais de compression quasiisentropique au moyen du MIVAR /13/, ainsi que des mesures de température résiduelle /14/, afin de discriminer entre les rôles respectifs de l'augmentation d'entropie et du retard à la transition traduit par le paramètre $\mathrm{A}$.

\section{IV - CONCLUSION}

L'objectif du travail présenté dans cet article était de développer un modèle de transformation polymorphique du fer qui soit thermodynamiquement cohérent et qui reproduise le comportement de ce matériau jusqu'à une contrainte de $500 \mathrm{kbar}$. Le modèle a été élaboré à partir d'une part des nombreuses données expérimentales présentées dans la littérature et d'autre part des résultats d'essais d'impact de plaques dont la configuration expérimentale originale nous a permis d'observer la transformation polymorphique inverse du fer. Les comparaisons entre les diagrammes expérimentaux et les diagrammes calculés par simulation numérique ont montré que la chronométrie et la structure des ondes etaient restituées à la fois pour la transformation directe et pour la transformation inverse. Néanmoins, un écart significatif entre le calcul et l'expérience a été constaté pour la transformation inverse. Des expériences complémentaires sont en cours de réalisation pour caractériser plus précisément ce dernier processus. Le point le plus délicat de cette caractérisation concerne l'interprétation de l'hystérésis observé expérimentalement entre les transformations directes et inverses. Les résultats numeriques indiquent, en effet, que cet hystérésis n'est lié qu'à la production irréversible d'entropie au cours du cycle compression-détente et ne correspond donc pas à un "retard" des transformations directes et inverses par rapport à la ligne de transformation polymorphique. Plusieurs observations expérimentales ne sont pas en accord avec ce résultat et nous avons donc conclu que des expériences supplémentaires étaient nécessaires afin de lever le doute sur ce point.

\section{REFERENCES}

/1/ D. Bancroft, E.L. Peterson et S. Minshall, J. Appl. Phys., 27, 291 (1956)

12/ P.C. Johnson, B.A. Stein et R.S. Davis, J. Appl. Phys., 33, 557 (1962)

13/ L.M. Barker et R.E. Hollenbach, J. Appl. Phys., 45, 11 (1974)

14/ P.M. Giles, M.H. Longenbach et A.R. Marder, J. Appl. Phys., 42, 4290 (1971)

15/ H.K. Mao, W.A. Basset et T. Takahashi, J. Appl. Phys., 38, 272 (1967)

16/ Y. Horie, Ph. D. Thesis, Washington State University (1966)

17/ D.J. Andrews, J. Phys. Chem. Solids, 34 (1973)

/8/ J.W. Forbes, Ph. D. Thesis, Washington State University (1976)

19/ M. Cowperthwaite, Am. J. Phys., 34, 1025 (1966)

/10/ J.N. Johnson, D.B. Hayes et J.R. Asay, J. Phys. Chem. Solids, 35 (1974)

/11/ G.E. Duvall et R.A. Graham, Reviews of Modern Physics, 49, 3 (1977)

112/ J. Petit et J. Picard, Rapport CEG T87-54 (1987)

/13/ M. Pérez, Shock Wave in Condensed Matter, 751 (1990)

/14/ M. Pérez et P. De Caso, Journées Détonique, ETBS, Bourges (1991) 